\title{
Estado nutricional en pacientes con cáncer cervico-uterino al ingreso hospitalario
}

\section{Nutritional status in patients with cervical cancer at hospital admission}

\section{RESUMEN}

Existe un gran aumento de la frecuencia de desnutrición en pacientes con cáncer, sin embargo, la información disponible sobre la evaluación del estado de nutrición en pacientes con cáncer cervicouterino ( $\mathrm{CaCu}$ ) es limitada. Material y métodos: Estudio retrospectivo, observacional, transversal. Se realizó una revisión de expedientes clínicos, en los cuales, el estado nutricional fue evaluado con una valoración nutricional institucional que incluye parámetros antropométricos (peso, IMC, pliegue cutáneo tricipital (PCT), circunferencia media de brazo (CMB), \% masa grasa), bioquímicos (albúmina, hemoglobina, linfocitos) y dietéticos (consumo energético y proteico). Resultados: Se incluyeron 195 expedientes de pacientes con $\mathrm{CaCu}$. La frecuencia de los estadios tempranos fue de $51.8 \%$ y en esas pacientes existió una prevalencia de desnutrición determinada por la albúmina de $9.75 \%$ y de $25.13 \%$ de acuerdo a los linfocitos, incrementándose a $41.18 \%$ de desnutrición de acuerdo al PCT en estadios avanzados. Se encontraron correlaciones estadísticamente significativas entre el estadio clínico y la ingesta calórica ( $r=-0.3062 p<0.0001)$, la concentración de albúmina ( $r=$ -0.4732 $p<0.0001)$ y la circunferencia media de brazo ( $r=-0.2564 p=0.0003$ ). Conclusión: El estado nutricional en estadios tempranos del CaCu no presenta alteraciones, mientras que en estadios intermedios y avanzados la alteración del estado nutricional aumenta.

Palabras clave: Estado de nutrición; Cáncer cervicouterino; Cáncer ginecológico; Cáncer; Nutrición.

\footnotetext{
ABSTRACT

There is a high frequency of malnutrition in cancer patients; however, available information on the assessment of nutritional status in patients with cervical cancer is limited. Material and methods: We conducted a retrospective, observational, cross-sectional study. Clinical records were reviewed; nutritional status was evaluated with the institutional nutritional evaluation tool that includes anthropometric (weight, BMI, tricipital skin fold, medium arm circumference, fat mass \%), biochemical (albumin, hemoglobin, lymphocytes) and diet parameters (energy and protein consumption). Results: We included the records of 195 patients with cervical cancer.
}

Emigdio Jaimes ${ }^{1,2}$, Diana Yolanda Reyes ${ }^{1,2}$, Yazmin Lizeth Beltrán ${ }^{2}$, Arturo González ${ }^{3}$, Carlos Ervin López ${ }^{1}$ Eva María Gómez

1. Centro Oncológico Estatal ISSEMyM, Toluca, México. 2. Facultad de Medicina Universidad Autónoma del Estado de México. 3. Universidad del Valle de Toluca.

Autor de correspondencia: Emigdio Jaimes Alpizar. Centro Oncológico Estatal ISSEMyM. Av Solidaridad las Torres 101, Colonia del parque CP 50180 Toluca Estado de México. Tel Oficina: (722) 2766820 Ext 2840 E-mail: emigdio.jaimes@yahoo.com

Este trabajo fue recibido el 07 de agosto de 2017. Aceptado con modificaciones: 29 de julio de 2018. Aceptado para ser publicado: 06 de agosto de 2018.

The frequency of the early stage cancer was $51.8 \%$ and in those patients there was a prevalence of malnutrition, determined by albumin, of $9.75 \%$ and $25.13 \%$ according to the lymphocytes, increasing to $41.18 \%$ measured by tricipital skin fold among patients with advanced-stage cancer. Statistically significant correlations were found between clinical stage and caloric intake $(r=-0.3062 p<0.0001)$, albumin concentration $(r=-0.4732 p<0.0001)$ and mean arm circumference ( $r=-0.2564 p=0.0003)$. Conclusion: The nutritional status in early stages of $\mathrm{CaCu}$ does not present alterations, while in intermediate and advanced stages the alteration of nutritional status may increase.

Key words: Nutritional status; Cervical cancer; Gynecological cancer; Cancer; Nutrition. 


\section{INTRODUCCION}

El cáncer es una enfermedad crónica producida por alteraciones genéticas y epigenéticas que afectan a la proliferación, senescencia y muerte celular'; la causa directa es desconocida, sin embargo, existen varios factores de riesgo. En el caso del cáncer cervicouterino $(\mathrm{CaCu})$ el virus del papiloma humano (VPH) ha sido identificado como uno de los principales factores; existen estudios realizados en pacientes mexicanos en donde se identificó la presencia del ADN del VPH en $95.8 \%$ de los casos en el periodo 2000-2001², lo cual es similar a lo reportado por estudios anteriores en los que se identificaba el virus en el $99.8 \%$ de los casos en pacientes norteamericanos durante el año $1999^{3}$. El VPH posee proteínas virales (E6 y E7) que cooperan entre sí para inmortalizar a los queratinocitos al interactuar con proteínas reguladoras del crecimiento celular que se encargan de proteger el genoma durante el ciclo celular, produciendo así diversas mutaciones asociadas con el cáncer ${ }^{4}$. También se ha observado una relación directamente proporcional en personas con múltiples parejas sexuales o que iniciaron a tener relaciones en edades tempranas, y el riesgo a padecer una lesión intraepitelial con exposición al VPH que, por consecuente, incrementa posibilidad de desarrollar $\mathrm{CaCu}^{5}$. Adicionalmente se ha descrito que el riesgo de neoplasia cervical aumenta con el número de gestaciones siendo hasta cinco veces mayor en aquellas con más de 12 partos en comparación con quienes han tenido 3 o menos .

De acuerdo al Instituto Nacional de Estadística y Geografía (INEGI) en el 2015 el CaCu se situó como la segunda causa de muerte por tumor maligno en $\mathrm{México}^{7}$ mientras que a nivel mundial la Organización Mundial de la Salud (mediante el proyecto GLOBOCAN) reporta al $\mathrm{CaCu}$ como el cuarto tipo de cáncer más frecuente en mujeres con un estimado de 266.000 decesos en el 2012, destacando que la mayoría de los casos se presentaron en países en desarrollo ${ }^{8}$.

Se ha documentado que el cáncer impacta negativamente el estado nutricional y se ha estimado una incidencia de desnutrición del $40 \%$ al $80 \%$ dependiendo del tipo de tumor, localización, estadio clínico y tratamiento; para diagnosticar desnutrición en pacientes oncológicos será necesario considerar factores adicionales como estrés metabólico aumentado, el cual afectará las proteínas séricas como la albúmina por lo que no deberá ser considerada como indicador del estado nutricional por si sola ${ }^{9}$. En los estudios relacionados con la pérdida de peso en pacientes oncológicos se encontró que el $7.2 \%$ de la muestra tuvo una perdida mayor al $10 \%$ de su peso habitual mientras que la mayoría $(79.8 \%)$ presentó una pérdida menor al $5 \%{ }^{10}$. Un estado de desnutrición se asocia a una menor respuesta a la quimioterapia y a una peor tolerancia (aumento de la toxicidad), debido a que en este cuadro clínico existe disminución de proteínas circulantes lo que impide un adecuado ligamiento de los fármacos a éstas, alterando sus periodos de semivida y sus características farmacocinéticas ${ }^{11}$.
El objetivo de este estudio es conocer el estado nutrición de las pacientes con $\mathrm{CaCu}$, ya que en la actualidad no existe información suficiente al respecto.

\section{MATERIAL Y MÉTODOS}

Estudio retrospectivo, observacional, descriptivo, transversal, donde se revisaron expedientes de pacientes del Centro Oncológico Estatal ISSEMMYM con diagnóstico de $\mathrm{CaCu}$, que acudieron por primera vez a consulta de nutrición vírgenes de tratamiento oncológico en el periodo de enero 2011 a diciembre 2015.

Los datos se recopilaron de la hoja de evaluación nutricional implementada en el centro oncológico, donde se evaluaron parámetros antropométricos (peso, estatura, porcentaje de pérdida de peso, pliegue cutáneo tricipital (PCT), circunferencia media de brazo $(\mathrm{CMB})$, porcentaje de masa grasa obtenida mediante bioimpedancia eléctrica con ayuda del equipo TANITA ${ }^{\circledR}$ BC533 INNERSCAN, en donde para la interpretación del pliegue cutáneo tricipital se comparó con los percentiles de referencia en el mismo grupo de edad ${ }^{12}$, quedando de la siguiente manera: Magro-deplesión de masa grasa: 0 - $<5$ Masa magra debajo del promedio: $>5-<15$, Masa grasa promedio: $>15-<75$, Masa grasa arriba del promedio: $>75-<85$ Exceso de masa grasa: $>85$. Para el porcentaje de grasa no saludable: $<8 \%$, aceptable bajo: $9-23 \%$, aceptable alto: $24-31 \%$ obesidad: $>32 \%{ }^{12}$. La interpretación de la circunferencia media de brazo se realizó con los percentiles de referencia de acuerdo a la edad. Riesgo de desnutrición: $<5$, Normal: 5-95, Riesgo de obesidad o hipertrofia muscular: $>95^{12}$. El índice de masa corporal (IMC) se clasificó de acuerdo al criterio de la OMS: bajo peso $<18.5 \mathrm{~kg} / \mathrm{m}^{2}$, normal $=18.5$ a $24.9 \mathrm{~kg} / \mathrm{m}^{2}$, sobrepeso $=25$ a $29.9 \mathrm{~kg} / \mathrm{m}^{2}$; obesidad I= $30 \mathrm{a}$ $34.9 \mathrm{~kg} / \mathrm{m}^{2}$, obesidad II= 35 a $39.9 \mathrm{~kg} / \mathrm{m}^{2}$, obesidad III $>40$ $\mathrm{kg} / \mathrm{m}^{2}$. El porcentaje de pérdida de peso se estimó con base en el peso habitual y el peso al momento de la evaluación y se definió como grave si es $>2 \%$ en una semana, $>5 \%$ en un mes, $>7.5 \%$ en tres meses y $>10 \%$ en seis meses ${ }^{13}$.

Para la albúmina sérica se utilizó la siguiente clasificación; desnutrición grave $<2.1 \mathrm{~g} / \mathrm{dl}$, desnutrición moderada 2.1-2.7g/ dl, desnutrición leve <2.7-3.5g/dl, Normal 3-5-5.0 g/dl . La concentración de hemoglobina se clasificó de la siguiente manera: $>12.0 \mathrm{~g} / \mathrm{l}=\sin$ anemia, $11.0-11.9 \mathrm{~g} / \mathrm{l}=$ anemia leve, 8.0-10.9 g/l = anemia moderada, $<8.0 \mathrm{~g} / \mathrm{l}=$ anemia severa $^{14}$. En los linfocitos totales la clasificación utilizada fue la siguiente: Normal 1.5 - 4.0×10 $/ \mathrm{L}$, desnutrición energético - proteica leve 1.2 - $1.5 \times 10^{9} / \mathrm{L}$, desnutrición energético - proteica moderada 0.9 - $1.2 \times 109 / \mathrm{L}$, desnutrición energético - proteica grave $<0.9 \times 10^{9} / \mathrm{L}^{12}$. Los parámetros dietéticos se obtuvieron a través del recordatorio de 24 horas, donde se calculó el porcentaje de adecuación de energía y proteína de acuerdo a las recomendaciones emitidas por ESPEN para todos los pacientes con cáncer en sus guías: ESPEN guidelines on nutrition in cancer patients. Se tomó como consumo deficiente: $<90 \%$, adecuada: $90-110 \%$, exceso: $>110 \%$ para ambos $\operatorname{casos}^{15}$. Los estadios clínicos de CaCu se clasificaron de acuerdo a la FIGO (International Federation of Gynecology and Obstetrics) $2009^{16}$. 
El tamaño de la muestra se conformó con todos los expedientes de los pacientes ingresados en el periodo de enero 2011 a diciembre 2015 y que cumplieron con los siguientes criterios de inclusión: valoración nutricional completa, vírgenes de tratamiento oncológico, ser paciente de primer ingreso en el COE. Se excluyeron los expedientes de pacientes que estuvieron incompletos.

Se utilizó estadística descriptiva e inferencial para analizar la frecuencia de casos de cada estadio clínico, su distribución por grupos de edad, la media de las variables antropométricas, bioquímicas y dietéticas; se buscaron asociaciones entre variables categóricas mediante el coeficiente de correlación de Spearman con el programa SPSS versión 22, se tomó como significancia estadística $\mathrm{p}=<0.5$.

El presente estudio se realizó bajo los lineamientos de la Declaración de Helsinki, en trabajo con seres humanos y fue aprobado por el Comité de Enseñanza e investigación del Centro Oncológico Estatal ISSEMyM.

Tabla 1. Incidencia de CaCu por edad

\begin{tabular}{|lcc|}
\hline Edad & $\begin{array}{c}\text { Frecuencia } \\
\mathbf{n}\end{array}$ & $\begin{array}{c}\text { Porcentaje } \\
\mathbf{( \% )}\end{array}$ \\
\hline 21-30 años & 19 & 9.74 \\
31-40 años & 49 & 25.13 \\
41-50 años & 45 & 23.07 \\
51-60 años & 52 & 26.67 \\
61-70 años & 20 & 10.26 \\
Mayor o igual a 71 años & 10 & 5.13 \\
N & 195 & 100.00 \\
\hline
\end{tabular}

\section{RESULTADOS}

El total de expedientes de pacientes que ingresaron en el periodo de estudio fue de 700 , únicamente se incluyeron 195 expedientes que cumplieron con los criterios de inclusión. La muestra se constituyó mayoritariamente de mujeres 31 a 40 años (25.13\%), 41 a 50 años $(23.07 \%)$ y de 51 a 60 años (26.67\%) (Tabla 1). Los estadios que se presentaron con mayor frecuencia fueron el I (51.28\%) y el II (28.2\%) (Tabla 2).

El índice de masa corporal muestra la frecuencia de $44.10 \%$ de sobrepeso y el $23.83 \%$ de las pacientes cursó con algún grado de obesidad al momento de la primera valoración nutricional. Otros parámetros antropométricos como el $\mathrm{PCT}$, la CMB y el porcentaje de masa grasa presentaron tendencias similares. Los niveles de hemoglobina, linfocitos y proteínas totales se mantuvieron en rangos normales mayoritariamente, se identificaron pocos casos de valores indicativos de desnutrición (Tabla 3).

Tabla 3. Características generales de las pacientes Min (mínimo), Máx (máximo)

\begin{tabular}{|c|c|c|c|c|}
\hline Edad (años) & 47,7 & $\pm 12,9$ & 22,0 & 84,0 \\
\hline Peso (kg) & 62,2 & $\pm 11,7$ & 37,8 & 102,6 \\
\hline $\mathrm{IMC}\left(\mathrm{kg} / \mathrm{m}^{2}\right)$ & 27,6 & $\pm 6,66$ & 16,9 & 61,5 \\
\hline PCT $\left(\mathrm{mm}^{3}\right)$ & 23,8 & $\pm 9,48$ & 6,00 & 52,0 \\
\hline Masa grasa $(\%)$ & 32,2 & $\pm 9,17$ & 2,50 & 69,2 \\
\hline Albúmina (g/dl) & 4,19 & $\pm 0,58$ & 1,70 & 6,70 \\
\hline Hemoglobina (g/dl) & 13,0 & $\pm 2,44$ & 4,40 & 17,7 \\
\hline Linfocitos $(4,0 \times 109 / \mathrm{L})$ & 2,61 & $\pm 3,07$ & 0,20 & 32,0 \\
\hline Consumo proteico $(\%)$ & 135,9 & $\pm 54,0$ & 8,0 & 356,0 \\
\hline
\end{tabular}

Min (mínimo), Máx (máximo)

Tabla 2. Clasificación de las pacientes por estadios clínicos.
\begin{tabular}{|lrc|}
\hline Estadio & $\mathbf{n}$ & $\%$ \\
\hline \multirow{2}{*}{ I } & 100 & $(51.28)$ \\
II & 55 & $(28.2)$ \\
III & 23 & $(11.79)$ \\
IV & 17 & $(8.71)$ \\
n & 195 & $(100.00)$ \\
\hline
\end{tabular}


Tabla 4. Prevalencia de desnutrición de acuerdo a parámetros nutricionales.

\begin{tabular}{|c|c|c|c|c|c|}
\hline Parámetro nutricional & $\begin{array}{l}\text { Prevalencia de } \\
\text { desnutrición } \\
\text { (General) }\end{array}$ & $\begin{array}{c}\text { Prevalencia de } \\
\text { desnutrición } \\
\text { (Estadio I) }\end{array}$ & $\begin{array}{c}\text { Prevalencia de } \\
\text { desnutrición } \\
\text { (Estadio II) }\end{array}$ & $\begin{array}{l}\text { Prevalencia de } \\
\text { desnutrición } \\
\text { (Estadio III) }\end{array}$ & $\begin{array}{r}\text { Prevalencia de } \\
\text { desnutrición } \\
\text { (Estadio IV) }\end{array}$ \\
\hline Índice de masa corporal <18,5kg/m2 & $2,05 \%$ & $0,97 \%$ & $3,77 \%$ & $0 \%$ & $5,88 \%$ \\
\hline $\begin{array}{l}\text { Pérdida de peso grave } \\
>2 \% \text { en una semana, } \\
>5 \% \text { en un mes } \\
>7,5 \% \text { en tres meses } \\
>10 \% \text { en seis meses, }\end{array}$ & $15,9 \%$ & $13,59 \%$ & $13,21 \%$ & $27,27 \%$ & $23,53 \%$ \\
\hline Pliegue cutáneo tricipital Percentil $<5$ & $25,13 \%$ & $16,5 \%$ & $28,3 \%$ & $45,45 \%$ & $41,18 \%$ \\
\hline$\%$ de masa grasa $<8 \%$ & $14,35 \%$ & $27,27 \%$ & $24,52 \%$ & $31,82 \%$ & $23,52 \%$ \\
\hline Albúmina $<3,5 \mathrm{~g} / \mathrm{dl}$ & $9,75 \%$ & $2,91 \%$ & $5,66 \%$ & $31,82 \%$ & $35,29 \%$ \\
\hline Hemoglobina $<12 \mathrm{~g} / \mathrm{l}$ & $26,67 \%$ & $7,76 \%$ & $33,95 \%$ & $63,64 \%$ & $70,58 \%$ \\
\hline Linfocitos totales $<1,5 \times 109 / \mathrm{L}$ & $24,62 \%$ & $17,47 \%$ & $32,07 \%$ & $31,83 \%$ & $35,26 \%$ \\
\hline Recomendación calórica <90\% & $22,05 \%$ & $15,53 \%$ & $24,53 \%$ & $36,36 \%$ & $35,29 \%$ \\
\hline
\end{tabular}

En la evaluación dietética se encontró que el 22.05\% de pacientes presentaron una ingesta hipocalórica e hipoproteica de forma general, en estadios tempranos (I y II) se identificó $15.3 \%$ y $24.53 \%$ de pacientes respectivamente mientras que en estadio III las dietas hipocalóricas e hipoproteicas incrementaron a $36.36 \%$ y en estadio IV a $35.29 \%$ (Tabla 4 ).

Después de evaluar el estado nutricional transversalmente, se ordenaron las variables de manera cualitativa ordinal para encontrar las asociaciones entre el estadio clínico con los parámetros nutricionales para buscar los parámetros más afectados cuando la enfermedad progresa, se utilizó correlación de Spearman para su análisis. En la tabla 5 se enlistan las asociaciones encontradas.

Se analizó la relación que existía entre el estadio clínico y cada una de las variables, utilizando correlación de Spearman. Se encontró que la edad tiene una correlación positiva siendo esta de manera significativa $(p=0.0034)$. En cuanto a medidas antropométricas se encontró una correlación negativa estadísticamente significativa, peso $(\mathrm{p}=$ $0.0028), C M B(p=0.0003)$, PCT $(p=0.0243)$. En el análisis de las pruebas bioquímicas encontramos una correlación negativa estadísticamente significativa con la albúmina ( $\mathrm{p}=$ $0-0001)$ y hemoglobina $(p=0.0001)$. En cuanto a parámetros dietéticos se encontró que existe que una correlación negativa en cuanto al consumo energético $(p=0.0001)$ y proteico $(p=0.0038)$, siendo estadísticamente significativa (Tabla 5).

\section{DISCUSIÓN}

La frecuencia de desnutrición que se encontró de acuerdo a diferentes parámetros nutricionales oscila entre 9.75 a $25.13 \%$ y hasta un $41.18 \%$ en estadios avanzados, siendo muy similar a lo reportado en la literatura donde la esta prevalencia está presente en un 15 a 20\% en el momento del diagnóstico ${ }^{17,18}$. Sin embargo, los resultados de esta investigación pudieran deberse a que la gran mayoría de las pacientes presentaban estadios tempranos de la enfermedad donde aún no existe alteración en el estado nutricional, solo un $20.52 \%$ tenían un estadio mayor a III. La concentración de hemoglobina se encontró disminuida en un $26.67 \%$. Sin embargo, no se consideró como parámetro de deficiencia debido a que muchas pacientes experimentan sangrado vaginal alterado. 
Tabla 5. Análisis de correlación del estadio clínico con las variables en estudio.

\begin{tabular}{|lcc|}
\hline Variable & $\mathbf{r}$ & $\mathbf{P} *$ \\
\hline Edad & 0.2086 & 0.0034 \\
Peso & -0.2127 & 0.0028 \\
IMC & -0.1211 & 0.0917 \\
Porcentaje de pérdida de peso & -0.0431 & 0.5496 \\
PCT & -0.1613 & 0.0243 \\
CMB & -0.2564 & 0.0003 \\
Porcentaje de masa grasa & -0.1056 & 0.1418 \\
Albúmina & -0.4732 & $<0.0001$ \\
Hemoglobina & -0.4788 & $<0.0001$ \\
Linfocitos & -0.1737 & 0.0152 \\
Consumo energético & -0.3062 & $<0.0001$ \\
Consumo proteico & -0.2066 & 0.0038 \\
\hline
\end{tabular}

${ }^{*} \mathrm{p}<0.05$ fue considerada estadísticamente significativa y se utilizó correlación de Spearman.

En el año $2009^{19}$ se realizó un estudio acerca de la anemia y su relación con el consumo alimentario y marcadores bioquímicos en pacientes con $\mathrm{CaCu}$, dicho estudio fue conformado por pacientes del año 2008 con diagnósticos de CaCu en estadios II y III que aún no recibían tratamiento oncológico; los resultados fueron que el $73 \%$ de las pacientes presentaban algún grado de anemia, no siendo así en el presente estudio donde la mayoría de las pacientes presentaban estadios tempranos. La pérdida de peso grave fue mayor en aquellas pacientes con estadios III y IV, incluso se puede observar que fue casi el dos veces más. En la gran mayoría de los parámetros podemos observar la tendencia a mayor desnutrición conforme el estadio avanza.

Dentro de las principales asociaciones que se encontraron fue que en estadio/edad se observa que conforme la mayoría de las pacientes con estadios avanzados eran de mayor edad, no podemos deducir a que se debe dicha causa, pero muy probablemente esté ligado a cuestiones socioculturales, creencias, falta de autocuidado de la salud y pobre acceso a servicios de salud, recordando que el $\mathrm{CaCu}$ se presenta en niveles socioeconómicos bajos ${ }^{20}$ y la población adulta es más vulnerable. La correlación estadio/peso, indica que, a mayor gravedad del estadio, menor será el peso del paciente. La relación entre el estadio/albúmina es negativa y significativa confirmando que, a mayor gravedad del estadio, menores serán los niveles de albúmina; a pesar de que no se midieron factores inflamatorios, estos dos últimos parámetros nutricionales se ven afectados por la misma evolución de la enfermedad debido a la elevación de proteínas proinflamatorias dando lugar a estos cuadros clínicos ${ }^{21}$.

En el caso de la relación entre el estadio/PCT y estadio/ $C M B$ se encuentran valores que sugieren una relación negativa significativa proponiendo que conforme aumenta la gravedad de la enfermedad disminuye la reserva grasa y muscular. No se encontraron estudios que utilizaran PCT y sus percentiles de referencia por edad como indicador de masa grasa o CMB con sus tablas de referencia como indicador para masa muscular por lo que no es posible comparar los resultados de dichas variables en este estudio.

La correlación estadio/consumo energético proveniente del recordatorio de 24 horas muestra una relación negativa significativa, esto proporciona la confirmación de que a medida que avanza el estadio, el consumo energético disminuye, lo cual se puede relacionar a la sintomatología derivada de estadios avanzados, hay que recordar que en este estudio también se encontró una relación negativa significativa entre la edad y el estadio por lo que existe la posibilidad de que pacientes de mayor edad con estadios avanzados sufran de más complicaciones para consumir alimentos comprometiendo tanto el consumo energético y proteico, ya que también se observó el mismo comportamiento en el consumo de proteínas.

Para comparar los resultados de este estudio se revisó bibliografía anterior sobre el estado nutricional en $\mathrm{CaCu}$ sin embargo hasta el momento no existe alguno que analice la relación entre los diferentes estadios clínicos y las variables antropométricas, bioquímicas, clínicas y dietéticas que permiten determinar el estado de nutrición.

Una de las limitaciones del estudio podría ser la ausencia de pacientes con estadio IA2 y IIA2, debido a que varios expedientes de pacientes ingresados durante el periodo de este estudio no cumplían con los criterios de inclusión al no contar con la información necesaria en su expediente o por haber recibido tratamiento oncológico previo al ingreso al COE.

\section{CONCLUSIONES}

La frecuencia de desnutrición encontrada en la muestra total de acuerdo a la albúmina sérica fue del 9.75\%. De acuerdo a los parámetros de linfocitos se encontró un $25.13 \%$ de desnutrición, mientras que en pacientes con CaCu en estadio IV se observó que $41.18 \%$ presento desnutrición de acuerdo con el PCT. La muestra fue constituida principalmente por pacientes de primer ingreso con estadio IA que presentaban indicadores antropométricos con valores clasificados como normales, sobrepeso y algún grado de obesidad sin pérdida de peso significativa ni valores bioquímicos alterados, sin embargo, se observó que 7 de las 10 variables presentaban alteraciones conforme el estadio avanzaba, siendo la ingesta calórica, los niveles de albúmina y disminución de la circunferencia media de brazo los que mostraron mayor asociación ( $p<0.05$ ).

Es importante brindar una correcta y oportuna atención nutricional en las pacientes con $\mathrm{CaCu}$ a fin de mejorar su estado nutricional durante el tratamiento antineoplásico.

Agradecimientos. A las autoridades del Centro Oncológico Estatal ISSEMYM por la autorización de este proyecto.

\section{BIBLIOGRAFÍA}

1. Gaillard S, Maxwell G, Sood A, Berchuck A. Biology and Genetics. In: Beret J, Hacker N. Gynecological oncology of 
Berek and Hacker. España: Wolters Kluwer. 2010. p. 2-36.

2. Tirado-Gómez LL, Mohar-Betancourt A, López-Cervantes $M$, García-Carrancá A, Franco-Marina F, Borges G. Risk factors for invasive cervical cancer in Mexican women. Public health Méx 2005; 47(5): 342-350.

3. Walboomers JM, Jacobs MV, MM Hands, Bosch FX, Kummer JA, Shah KV, et al. Human papillomavirus is a necessary cause of invasive cervical cancer worldwide. J Pathol 1999; 189(1): 12-9.

4. Shell M. Diagnosis and therapy of human papillomavirus. Rev Chil Infect 2007; 24(3): 209-214.

5. Ortiz Serrano R, Uribe Pérez CI, Díaz Martínez LA, Yuriko R. Risk factors for cervical cancer. Rev Colomb Obstet Ginecol 2004; 55(2): 146-160.

6. Castañeda MS, Toledo R, Aguilera M. Risk factors for cervical cancer in Zacatecas women. Public Health of Mexico 1998; 40(3): 30-338.

7. INEGI. Statistics regarding the day of cancer 2016 [Cited on 06/21/16]. Available at: http://www.inegi.org.mx/saladeprensa/ aproposito/2016/cancer2016_0.pdf

8. WHO, GLOBOCAN 2012: Estimated Cancer Incidence, Mortality and Prevalence Worldwide in 2012 [Cited 2017 Jun 01]. http://globocan.iarc.fr/Pages/ fact_sheets_cancer.aspx

9. Martínez R. Assessment of nutritional status in cancer patients. INCAN [Internet] 2007 [Quoted 2017 Jun 01]; 2: 315-26. http://incan-mexico.org/revistainvestiga/elementos/ documentosPortada/1207758983.pdf

10. Dufau L. Prevalence of malnutrition in cancer patients. Diaeta [Internet]. 2010 Mar [Quoted 2017 Jun 01]; 28 (130): 31-36. http:// www.scielo.org.ar/scielo.php?script= sci_arttext\&pid=S185273372010000100006\&lng=en.

11. Murry DJ, Riva L, Poplack DG. Impact of nutrition on pharmacokinetics of antineoplastic agents. Int I Cancer Suppl 1998; 11: 48-51.

12. Suverza A, Haua K. The ABCD of the evaluation of the state of nutrition. Mexico, D. F: McGraw Hill 2010. p. 3-10, 29-68, 173-177, 258-266.

13. Official Mexican NOM-043-SSA2-2012, Basic Health Services. Promotion and education for health in alimentary matters. Criteria for guidance.

14. WHO. Haemoglobin concentrations for the diagnosis of anaemia and assessment of severity. Vitamin and Mineral Nutrition Information System. Geneva, World Health Organization, 2011 (WHO/NMH/NHD/MNM/11.1). http://www.who.int/ vmnis/ indicators/haemoglobin.pdf

15. Arends J, Bachmann P, Baracos V, Barthelemy N, Bertz H, Bozzetti $F$, et al. ESPEN guidelines on nutrition in cancer patients. Clin Nutr 2017; 36(1): 11-48.

16. Diagnosis and treatment of cervical cancer. Mexico: Secretary of Health, 2010. http://www.cenetec.salud.gob.mx/descargas/ gpc/CatalogoMaestro/433_GPC_Ca_cervicouterino/GER_ Cxncer_cervicouterino.pdf

17. Fernández López MT, Saenz Fernández CA, De Sás Prada MA, Alonso Urrutia S, Bardasco Alonso ML, Alves Pérez MT, et al. Malnutrition in cancer patients; An experience of four years. Nutr Hosp 2013; 28(2): 372-381.

18. Cid Conde L, Fernández López T, Neira Blanco P, Arias Delgado J, Varela Correa JJ, Gómez Lorenzo FF. Prevalence of malnutrition in patients with digestive neoplasia prior to surgery. Nutr Hosp 2008; 23(1): 46-53.

19. Bichara FE, Amancio O, Gutiérrez-Salmeán G, Fuchs V. Determination of the type of anemia and its relation with food intake and biochemical markers in patients with cervical cancer. Rev Chil Nutr 2009; 36(4): 1074-1079.

20. Faggiano F, Partanen T, Kogevinas M, Boffetta P. Socioeconomic differences in cancer incidence and mortality. IARC Sci Publ 1997; 138: 65-176.

21. Argilés JM, Busquets S, López- Soriano FJ, Figueras $M$. Pathophysiology of neoplastic cachexia. Nutr Hosp 2006; 21 (Suppl 3) 4-9. 